\title{
A study protocol for a randomised crossover study evaluating the effect of diets differing in carbohydrate quality on ileal
}

\section{content and appetite regulation in healthy humans [version 1;}

\section{peer review: 2 approved with reservations]}

\section{Claire S. Byrne ${ }^{1} 1$, Dominic Blunt 2 , James Burn², Edward Chambers',} Aygul Dagbasi ${ }^{1}$, Georgia Franco Becker¹, Glenn Gibson³, Lilian Mendoza1, Kevin Murphy4, Carlos Poveda3 ${ }^{3}$ Anya Ramgulam4, Martina Tashkova1, Gemma Walton ${ }^{3}$, Chaiwat Washirasaksiri', Gary Frost (iD1

\footnotetext{
${ }^{1}$ Section for Nutrition Research, Department of Medicine, Imperial College London, London, UK

${ }^{2}$ Department of Imaging, Charing Cross Hospital, Imperial NHS Trust, London, UK

${ }^{3}$ Department of Food and Nutritional Sciences, University of Reading, Reading, UK

${ }^{4}$ Section of Endocrinology and Investigative Medicine, Department of Medicine, Imperial College London, London, UK
}

V1 First published: 05 Mar 2019, 8:258

https://doi.org/10.12688/f1000research.17870.1

Latest published: 22 Oct 2019, 8:258

https://doi.org/10.12688/f1000research.17870.2

\section{Abstract}

Introduction: A major component of the digesta reaching the colon from the distal ileum is carbohydrate. This carbohydrate is subject to microbial fermentation and can radically change bacterial populations in the colon and the metabolites they produce, particularly short-chain fatty acids (SCFA). However, very little is currently known about the forms and levels of carbohydrate in the ileum and the composition of the ileal microbiota in humans. Most of our current understanding of carbohydrate that is not absorbed by the small intestine comes from ileostomy models, which may not reflect the physiology of an intact gastrointestinal tract.

Methods: We will investigate how ileal content changes depending on diet using a randomised crossover study in healthy humans.

Participants will be inpatients at the research facility for three separate 4-day visits. During each visit, participants will consume one of three diets, which differ in carbohydrate quality: 1) low-fibre refined diet; 2) high-fibre diet with intact cellular structures; 3) high-fibre diet where the cellular structures have been disrupted (e.g. milling, blending). On day 1, a nasoenteric tube will be placed into the distal ileum and its position confirmed under fluoroscopy. Ileal samples will be collected via the nasoenteric tube and metabolically profiled, which will determine the amount and type of carbohydrate present, and the composition of the ileal microbiota will be measured. Blood samples

\section{Open Peer Review \\ Approval Status \\ 1 2 \\ version 2 \\ (revision) \\ 22 Oct 2019 \\ version 1 \\ 05 Mar 2019

$\begin{array}{cc} & \\ \text { view } & \text { view } \\ ? & ? \\ \text { view } & \text { view }\end{array}$ \\ 1. Ellen E. Blaak ID), Maastricht University Medical Centre, Maastricht, The Netherlands \\ 2. Marcel van de Wouw, University College \\ Cork, Cork, Ireland \\ Harriët Schellekens (iD), University College \\ Cork, Cork, Ireland \\ University College Cork, Cork, Ireland}

Any reports and responses or comments on the article can be found at the end of the article. 
will be collected to assess circulating hormones and metabolites. Stool samples will be collected to assess faecal microbiota composition. Subjective appetite measures will be collected using visual analogue scales. Breath hydrogen will be measured in real-time as a marker of intestinal fermentation. Finally, an in vitro continuous fermentation model will be inoculated with ileal fluid in order to understand the shift in microbial composition and SCFA produced in the colon following the different diets.

Registration: ISRCTN11327221.

Keywords

Dietary Fibre, Carbohydrate, Gastrointestinal tract, Ileum, Colon, Gut microbiota, Nasoenteric

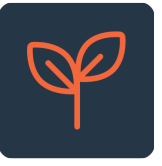

This article is included in the Agriculture, Food and Nutrition gateway.

Corresponding author: Gary Frost (g.frost@imperial.ac.uk)

Author roles: Byrne CS: Conceptualization, Investigation, Methodology, Project Administration, Supervision, Writing - Original Draft Preparation, Writing - Review \& Editing; Blunt D: Conceptualization, Investigation, Supervision, Writing - Review \& Editing; Burn J: Investigation, Writing - Review \& Editing; Chambers E: Methodology, Writing - Review \& Editing; Dagbasi A: Conceptualization, Investigation, Methodology, Project Administration, Supervision, Writing - Original Draft Preparation, Writing - Review \& Editing; Franco Becker G: Investigation, Writing - Review \& Editing; Gibson G: Conceptualization, Funding Acquisition, Methodology, Writing - Review \& Editing; Mendoza L: Investigation, Writing - Review \& Editing; Murphy K: Conceptualization, Funding Acquisition, Methodology, Writing - Review \& Editing; Poveda C: Investigation, Writing - Review \& Editing; Ramgulam A: Investigation, Writing - Review \& Editing; Tashkova M: Investigation, Writing - Review \& Editing; Walton G: Conceptualization, Investigation, Methodology, Writing - Review \& Editing; Washirasaksiri C: Investigation, Writing - Review \& Editing; Frost G: Conceptualization, Funding Acquisition, Methodology, Project Administration, Supervision, Writing - Review \& Editing

Competing interests: No competing interests were disclosed.

Grant information: This article presents independent research funded by the UK Biotechnology \& Biological Sciences Research Council (BBSRC) (BB/N016847/1) and Nestec Ltd, and is supported by the NIHR CRF and BRC at Imperial College Healthcare NHS Trust. The views expressed are those of the authors and not necessarily those of our funders, the NHS, the NIHR or the Department of Health. The Section of Endocrinology and Investigative Medicine is funded by grants from the MRC, BBSRC, NIHR, an Integrative Mammalian Biology (IMB) Capacity Building Award, an FP7- HEALTH- 2009- 241592 EuroCHIP grant and is supported by the NIHR Biomedical Research Centre Funding Scheme. GF holds an NIHR Senior Investigator Award.

The funders had no role in study design, data collection and analysis, decision to publish, or preparation of the manuscript.

Copyright: $\odot 2019$ Byrne CS et al. This is an open access article distributed under the terms of the Creative Commons Attribution License , which permits unrestricted use, distribution, and reproduction in any medium, provided the original work is properly cited.

How to cite this article: Byrne CS, Blunt D, Burn J et al. A study protocol for a randomised crossover study evaluating the effect of diets differing in carbohydrate quality on ileal content and appetite regulation in healthy humans [version 1; peer review: 2 approved with reservations] F1000Research 2019, 8:258 https://doi.org/10.12688/f1000research.17870.1

First published: 05 Mar 2019, 8:258 https://doi.org/10.12688/f1000research.17870.1 


\section{Introduction}

Obesity

Obesity is a chronic health problem that has reached epidemic proportions globally ${ }^{1}$. Obesity increases the risk of developing a range of non-communicable diseases including type 2 diabetes, cardiovascular disease, certain cancers and osteoarthritis ${ }^{2}$. It has been projected that obesity rates could double by 2050 , which would add $£ 5.5$ billion to the total annual expenses of the National Health Service (NHS) ${ }^{3}$. The Foresight report highlighted appetite regulation as a major target in the dietary treatment of obesity ${ }^{3}$. However, it is not yet fully understood how the gastrointestinal (GI) tract senses dietary content in order to suppress subsequent food intake. This knowledge could inform the development of foods or dietary regimes that increase fullness and prevent weight gain ${ }^{4}$.

\section{The gastrointestinal tract}

The GI tract is the largest endocrine organ in the body and is responsible for digesting and absorbing dietary components. The GI tract is also the host to a microbiota, which ferment undigested material. The GI tract senses changes in the luminal nutrient content and modulates neuronal and hormonal signals from the GI tract in order to help regulate appetite and food intake $^{5}$. The anorexigenic gut hormones peptide YY (PYY) and glucagon-like peptide-1 (GLP-1), released from enteroendocrine cells $(\mathrm{EEC})^{5}$, are examples of such GI signals secreted following a meal in a two-phase process. The first phase is thought to be mediated primarily by neural mechanisms and may help drive satiation (the sum of processes that cause meal termination). The second phase is thought to be mediated by direct nutrient sensing in the lower parts of the GI tract and to be an important long-term satiety signal. The peripheral administration of PYY or GLP-1 has previously been shown to reduce food consumption in animal models and humans, highlighting the importance of these gut hormones ${ }^{6-10}$. It has therefore been suggested that the incorporation into the diet of foods that stimulate a greater gut hormone secretion could suppress appetite to a greater extent and therefore help control body weight in the long term.

\section{Dietary fibre}

There is an increasing amount of evidence to suggest that consumption of dietary fibre is beneficial to human health. Dietary fibre refers to carbohydrates that cannot be digested by mammalian enzymes, and thus remain in relatively intact form when they reach the caecum, where they are subsequently available for fermentation by the gut microbiota. Epidemiological evidence suggests that diets high in non-digestible fibres are associated with lower body weight gain in humans ${ }^{11,12}$. In addition, fermentable fibres have been shown to protect against weight gain and fat mass development in rodents fed a high-fat diet ${ }^{13}$. Dietary fibre is thought to aid in weight management through a number of mechanisms including the promotion of satiation, increased GI transit time and stimulation of gut hormone secretion ${ }^{14}$. Short-chain fatty acids, products of microbial fermentation, have also been shown to stimulate the secretion of GLP-1 and PYY. Together, this evidence suggests that fibre may be beneficial in the management of obesity.

\section{Importance of the cellular structure of foods}

The cellular structure of foods can also be an important determinant of the subsequent impact of a food on appetite. In order for macronutrients within foods to be digested, they need to be in contact with digestive enzymes of the GI tract. In plant tissues, cell wall rupture can lead to a release of macronutrients into the extracellular environment, or enzymes can diffuse through a permeable cell wall in order to digest the encapsulated macronutrients ${ }^{15}$. However, cell wall matrices or individual cell wall polysaccharides of plant foods can behave in a variety of ways during digestion. For example, some cell walls are highly impermeable and less susceptible to rupture, which leads to a reduction in the rate and extent of nutrient release and digestion. Thus, the cell walls of plants can behave as a physical barrier to digestion in the upper GI tract. The degree of domestic and industrial processing (e.g. milling, blending) of plant foods and ingredients also affects macronutrient bioaccessibility and digestion by modifying the structural integrity of the plant tissue. The importance of cell wall integrity has previously been highlighted in determining the effect of plant foods on physiological functions. These studies reported that structurally intact plant tissues tend to be digested to a lesser extent and at a slower rate, which attenuated the postprandial rise in glycaemia and/or lipaemia ${ }^{16-18}$, and may also trigger the release of lower concentrations of anorexigenic gut hormones within the small intestine, compared to foods in which the nutrients are bioaccessible. However, upon reaching the colon from the distal ileum, undigested plant material serves as a substrate for microbial fermentation, which leads to the production of SCFA and triggers the release of anorexigenic gut hormones. This process is thought to result in a more long-term satiety signal following the consumption of minimally processed plant-based foods.

\section{Study rationale}

Within the GI tract, the colon contains the highest density of EEC $^{19}$. These cells express a large array of $\mathrm{G}$ protein coupled receptors (GPCRs) that sense nutrients and metabolites in the colonic lumen ${ }^{20}$. This gives the colon the ability to regulate appetite and the GI tract in response to the nutrient environment in the colonic lumen. A major component of the digesta reaching the colon is carbohydrate, which is a major fuel source of the gut microbiota ${ }^{21}$. The flow of carbohydrate into the colon from the distal ileum can radically change populations of bacteria in the colon and metabolites they produce, particularly SCFAs $^{21}$. However, very little is known about the type and amount of carbohydrate present in the ileum as well as the composition of the ileum microbiota in humans. Most of our current understanding of carbohydrate that is not absorbed by the small intestine comes from ileostomy models ${ }^{22}$. These models may not reflect the physiology of an intact GI tract, as the gut alters following the surgery. In the present study we will use an ileum intubation method in healthy humans, which will allow us to gain a 'real-time' perspective on how the actual ileum content changes in response to different diets and the subsequent effect on measures of appetite and gut hormone secretion. We will also conduct an in vitro study in order to understand how ileum content affects the colonic gut microbiota and the production of 
SCFA in the colon, as this is important in determining how dietary carbohydrate drives colonic gut hormone release.

\section{Study objectives}

Hypothesis

We hypothesise that the consumption of minimally-processed high-fibre foods will result in the presence of a greater number of intact cellular structures in the ileum, which will subsequently be fermented in the colon resulting in the production of SCFAs and greater release of PYY and GLP-1.

\section{Objectives}

There are a number of objectives to the present study: to identify the impact of dietary carbohydrate on (1) the forms and levels of carbohydrates in, and (2) the microbiological profile of, the ileum, and to (3) to determine whether the carbohydrate content of the ileum relates to appetite responses and gut hormone release.

\section{Primary and secondary outcome measures}

The co-primary outcome measures for this study are:

1. Metabolic profiling of ileal samples

2. Microbiological profiling of ileal samples

The secondary outcome measures include:

1. Microscopy of ileal samples for plant structures, cellular structures and starch granules

2. Metabolic and hormonal profiling of blood samples

3. Metabolic and microbiological profiling of faecal samples

4. Subjective appetite measurements

5. Breath $\mathrm{H}_{2}$ concentrations

6. Ileum samples for the inoculation of an in vitro continuous fermentation model

\section{Protocol}

This study will be a randomised crossover study consisting of three separate 4-day inpatient study visits at the National Institute for Health Research (NIHR) Imperial Clinical Research Facility (CRF), Hammersmith Hospital, London, United Kingdom.

\section{Health screening}

Healthy humans will be recruited using existing healthy volunteer databases (e.g. the Healthy Volunteer Panel at the NIHR Imperial CRF) and by advertisement in public buildings, on the internet and social media. Participants who express an interest in taking part in the study will be provided with the Patient Information Sheet (PIS). Following an initial telephone screening, potential participants will be invited to attend a health screening visit at the NIHR Imperial CRF in order to further assess their eligibility and provide written, informed consent to a member of the study team. The consent form and participant information sheet are available on Figshare $^{23}$. During health screening, the study protocol and the risks and benefits of participating will be explained in full and any questions that the participant may have will be answered. Participants will be asked questions about their medical history, anthropometric measurements will be collected, blood pressure (BP) will be measured, an echocardiogram (ECG) will be performed and a blood sample taken. The collected blood sample (1 x $20 \mathrm{ml})$ will be used to assess $\mathrm{HbA1c}$, full blood count, liver function, renal function and blood lipids. A pregnancy test will be performed on women of childbearing age. Inclusion and exclusion criteria will be assessed as described.

\section{Study population}

Healthy humans aged between 18-65 years (inclusive) with a body mass index (BMI) of $18.5-30 \mathrm{~kg} / \mathrm{m}^{2}$ will be recruited for this study. They must also show a willingness and ability to give written informed consent and to understand, to participate and to comply with the study requirements. Exclusion criteria includes: abnormal echocardiograph (ECG), screening blood results outside of normal reference values, weight change of $\geq 5 \mathrm{~kg}$ in the preceding 2 months, current smokers, history of substance abuse and/or excess alcohol intake in the last 2 years, pregnancy, diabetes, cardiovascular disease, cancer, gastrointestinal disease, kidney disease, liver disease, pancreatitis, started new medication within the last 3 months likely to interfere with energy metabolism/ appetite regulation/hormonal balance, antibiotic use within the last months, participation in a research study in the 12 week period prior to entering this study, any blood donation within the 12 week period prior to entering this study.

\section{Randomisation}

Following the health screening, eligible participants will be randomised into the study. Randomisation will be performed by an independent internet and telephone randomisation company (sealedenvelope.com). Due to the different physical appearance of the diets, participants will not be blinded.

\section{Sample size}

We aim to recruit 15 subjects. This is a pilot study in a new area of research and therefore a formal power calculation is not possible. However, a recent study in ileostomy patients investigating differences in the carbohydrate output in ileal effluent depending on the quality of carbohydrate consumed was able to detect significant differences between outcome measures in a similar number of subjects $(n=9)^{17}$.

\section{Dietary intervention}

Dietary calculations. All three diets are tailored to meet the energy requirements (TEE, total energy expenditure) of each participant. Using the participant's weight and age at screening, Schofield equations will be used to calculate each participant's basal metabolic rate (BMR) as shown in Table 1. This will then be multiplied by 238.85 to convert $\mathrm{MJ} / \mathrm{d}$ to $\mathrm{kcal} / \mathrm{d}$ and then by 1.2 to account for their low physical activity level (PAL) while being inpatients at the CRF.

Dietary overview. During the 3 separate study visits, volunteers will be provided with diets differing in carbohydrate quality, 
Table 1. Formula for calculating total energy expenditure (TEE) (kcal/day) using the Schofield equations and physical activity level correction.

\begin{tabular}{|l|l|l|l|}
\hline Gender & Age range $(\mathbf{y})$ & Regression formula for BMR (MJ/d) & TEE calculation $(\mathbf{k c a l} / \mathbf{d})$ \\
\hline Male & $18-29$ & $0.063 \times$ weight $(\mathrm{kg})+2.896$ & * 238.85 (To convert MJ to kcal) * $1.2(\mathrm{PAL})$ \\
\hline & $30-59$ & $0.048 \times$ weight $(\mathrm{kg})+3.653$ & \\
\hline & $60-74$ & $0.0499 \times$ weight $(\mathrm{kg})+2.930$ & \\
\hline Female & $18-29$ & $0.062 \times$ weight $(\mathrm{kg})+2.036$ & \\
\hline & $30-59$ & $0.034 \times$ weight $(\mathrm{kg})+3.538$ & \\
\hline $60-74$ & $0.0386 \times$ weight $(\mathrm{kg})+2.875$ & \\
\hline
\end{tabular}

BMR, basal metabolic rate.

PAL, physical activity level.

TEE, total energy expenditure.

which have been designed using DietPlan 6 software. In a randomised order, volunteers will receive:

- Diet 1 (low fibre (LF)-refined): This diet contains highly refined and processed carbohydrate. Foods are low in fibre and intact cell structures.

- Diet 2 (high fibre (HF)-whole): This diet is high in fibre providing a high intake of intact cellular structures. Foods have resistant cell structures such as beans, nuts, fruit and vegetables.

- Diet 3 (HF-disrupted): This diet is also high in fibre but with disrupted cellular structures. It is the same as the HF-whole diet, but foods will be milled or blended to disrupt the cellular structure.

All the diets have a similar macronutrient content (55\% energy from carbohydrate, $30 \%$ energy from fat, $15 \%$ energy from protein). However, the quality of carbohydrate present is significantly different. For example, the HF-whole and HF-disrupted diets contain $46.5 \mathrm{~g}$ and $47.7 \mathrm{~g}$ dietary fibre/2000 kcal, respectively, while the LF-refined diet contains only $16.3 \mathrm{~g}$ dietary fibre/2000 kcal.

Breakfast, lunch and dinner will each provide $30 \%$ of the participant's prescribed caloric intake and an evening snack will provide the final $10 \%$. The dietary plan for each diet is outlined in Table 2. All food provided to the participants will be prepared by the study team in the CRF Diet Kitchen according to food hygiene/food safety standards.

Diet preparation. For the LF-refined diet, breakfast will be prepared the night before serving but the bread will be toasted and the milk will be added to the cornflakes immediately before serving. The meals served at 13:00 and 17:00 will be ready meals, which will be cooked in the microwave according to the manufacturer's instructions and the required portion will be weighed out before serving.

For the HF-whole diet, the breakfast will be prepared the day before serving and the oats will be soaked in milk from approximately 16:30 the evening before. The lunch will be prepared in the hour before serving; the apples will be cut into chunks that are easier to eat and the soup will be weighed out and microwaved just before serving. The dinner will be prepared in the hour before serving. The potatoes will be boiled for $20 \mathrm{~min}$ and the peas microwaved according to the manufacturer's instructions. Both will be drained and weighed after cooking. All other foods will be served cold. The carrot, beetroot and tomatoes will be cut up into smaller pieces that are easier to eat. The evening snack will be prepared at dinner-time.

For the HF-disrupted diet, the oats will be blended in advance into a flour-like consistency. The rye bread and humous will be made in advance in-house, frozen and defrosted as needed. The breakfast will be prepared the day before serving. The oranges will be blended to a smoothie. The oats will be soaked in milk from approximately 16:30 the evening before and heated in the microwave immediately before serving. The lunch will be prepared in the hour before serving. The apples will be blended to a puree. The can of soup will be blended, the required portion will be weighed out and microwaved just before serving. The dinner will be prepared in the hour before serving. The beetroot and tomatoes will be blended to a smoothie. The potatoes and carrots will be boiled for $20 \mathrm{~min}$ and the peas will be microwaved according to the manufacturer's instructions. The vegetables will then be drained, blended and weighed. The potato, carrot and peas will then be re-heated in the microwave to comply with food safety standards. All other foods will be served cold. The snack is prepared at dinner-time and the bananas and oranges will be blended.

All foods that are heated or cooked by the study team will be temperature probed before serving.

\section{Study visit protocol}

An overview of the study visit protocol is outlined in Figure 1. Participants will attend their first study visit within 12 weeks of their screening visit. There will be a minimum wash out period of 7 days between study visits.

Day 1: Nasoenteric tube insertion. Participants will be asked to arrive at the Imaging Department in Charing Cross Hospital, London, having fasted overnight and having avoided intense exercise, caffeine and alcohol the day before their study visit. Females of child-bearing age will be asked to provide a urine 
Table 2. Dietary plan for intervention diets.

\begin{tabular}{|c|c|c|c|c|c|}
\hline Meal & Time & LF-refined & HF-whole & HF-disrupted & $\%$ E provided \\
\hline \multirow[t]{6}{*}{ Breakfast } & $9 \mathrm{am}$ & Cornflakes cereal & Jumbo oats & Finely milled oats, blended & 30 \\
\hline & & Whole milk & Whole milk & Whole milk & \\
\hline & & White bread, toasted & German style rye bread & Rye bread made with wholemeal flour & \\
\hline & & Margarine & Margarine & Margarine & \\
\hline & & Full fat natural yoghurt & Full fat natural yoghurt & Full fat natural yoghurt & \\
\hline & & & Oranges & Oranges, blended & \\
\hline \multirow[t]{5}{*}{ Lunch } & $1 \mathrm{pm}$ & Beef lasagne & $\begin{array}{l}\text { Carrot and butterbean soup } \\
\text { (tinned) }\end{array}$ & Carrot and butterbean soup (tinned), blended & 30 \\
\hline & & Coca Cola & German style rye bread & Rye bread made with wholemeal flour & \\
\hline & & & Margarine & Margarine & \\
\hline & & & Apples & Apples, blended & \\
\hline & & & Plain peanuts & Plain peanut butter & \\
\hline \multirow[t]{7}{*}{ Dinner } & $5 \mathrm{pm}$ & Shepherd's pie & Chicken breast (pre-packed) & Chicken breast (pre-packed) & 30 \\
\hline & & Coca Cola & Boiled new potatoes & Boiled new potatoes, blended & \\
\hline & & Wine gum sweets & Raw carrot & Boiled carrot, blended & \\
\hline & & & Peas (tinned) & Peas (tinned), blended & \\
\hline & & & Beetroot (pre-packed) & Beetroot (pre-packed), blended & \\
\hline & & & Cherry tomatoes & Cherry tomatoes, blended & \\
\hline & & & $\begin{array}{l}\text { Chickpeas* (tinned) with } \\
\text { lemon juice, olive oil, parsley, } \\
\text { mint, black pepper, salt }\end{array}$ & $\begin{array}{l}\text { Chickpea humus (Chickpeas (tinned) with } \\
\text { lemon juice, olive oil, parsley, mint, black } \\
\text { pepper, salt, which are blended into a humus) }\end{array}$ & \\
\hline \multirow[t]{4}{*}{ Snack } & $9 \mathrm{pm}$ & White bread & German style rye bread & Rye bread made with wholemeal flour & 10 \\
\hline & & Margarine & Margarine & Margarine & \\
\hline & & $\begin{array}{l}\text { Medium cheddar } \\
\text { cheese }\end{array}$ & Bananas & Bananas, blended & \\
\hline & & & Oranges & Oranges, blended & \\
\hline
\end{tabular}

${ }^{*}$ Chickpea and chickpea humus recipes will be energy matched. E, energy, HF, high fibre; LF, low fibre.

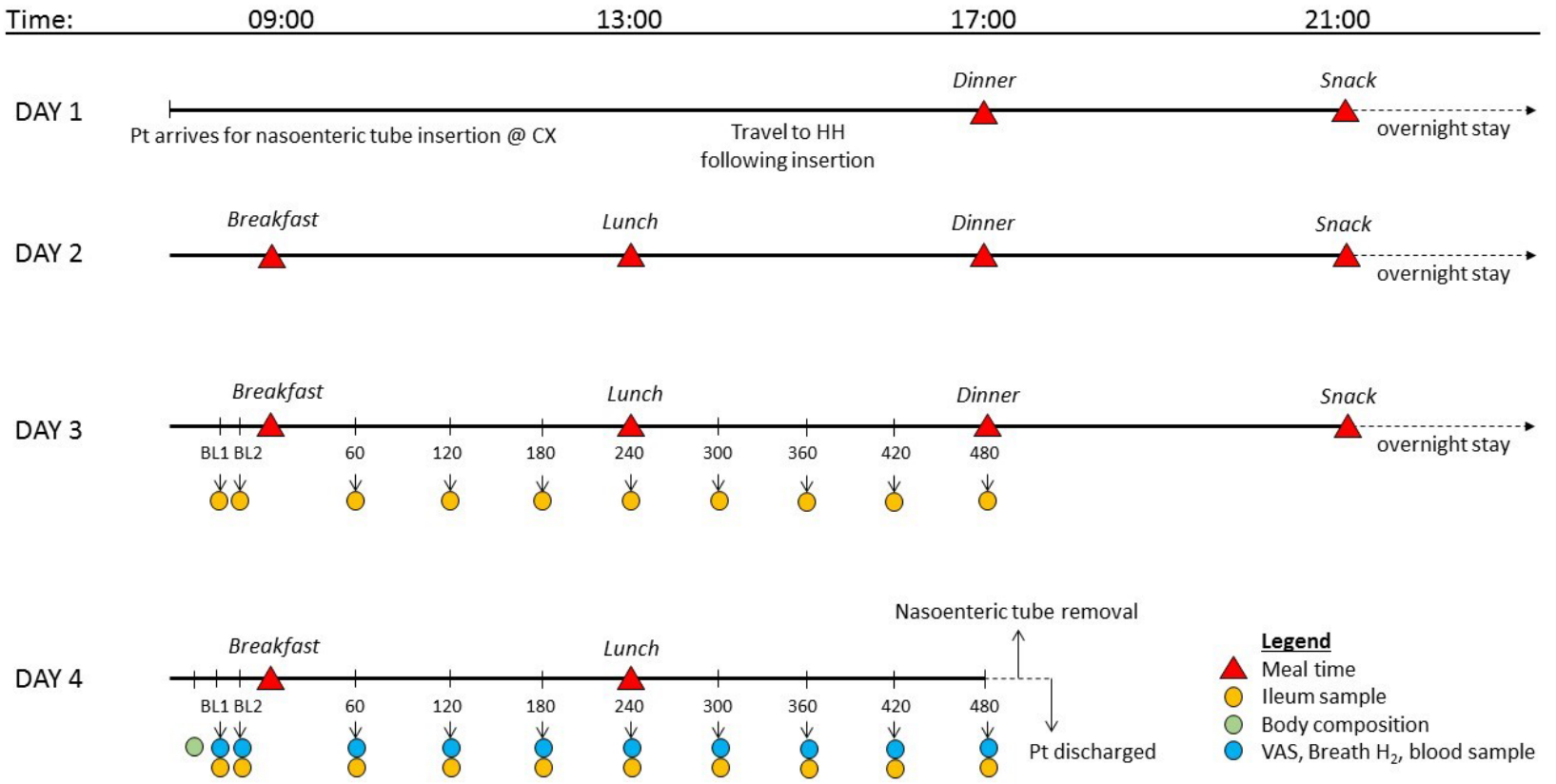

Figure 1. Study visit overview. Abbreviations: BL, baseline; CX, Charing Cross Hospital; HH, Hammersmith Hospital; Pt, patient; VAS, visual analogue scale. 


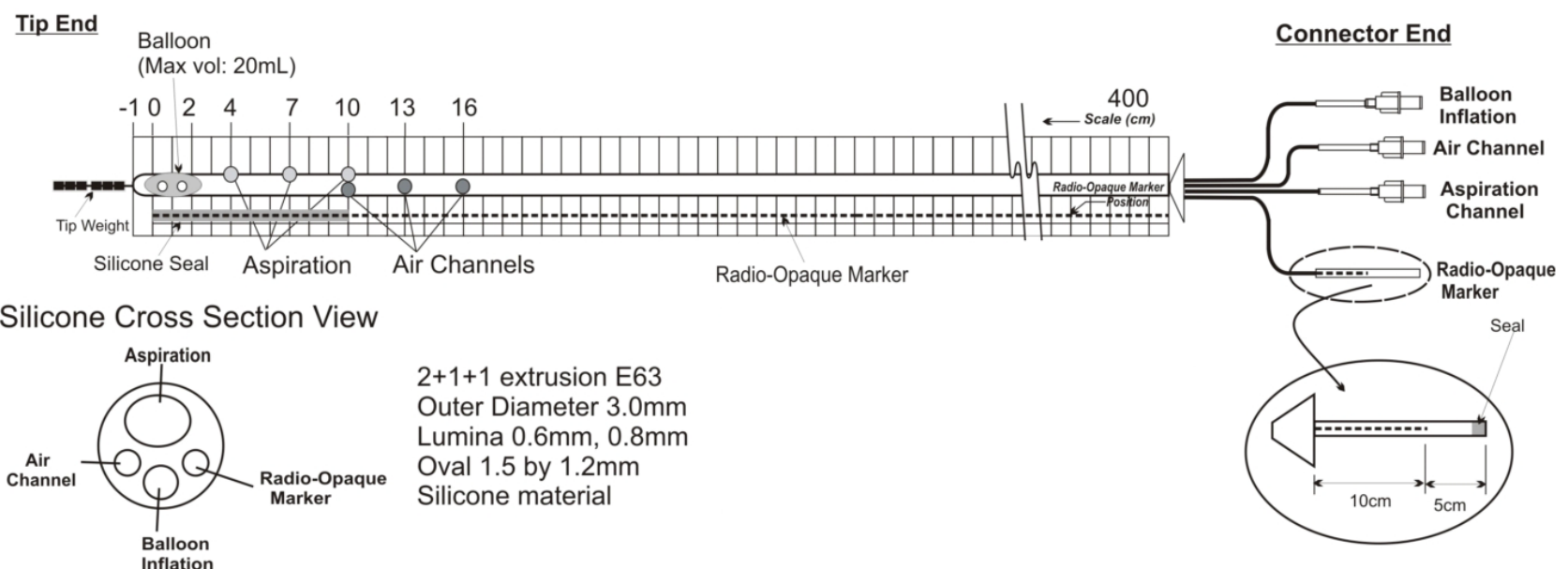

Figure 2. Nasoenteric tube design. Published with permission from MUI Scientific.

specimen in order to perform a pregnancy test. A custom-made nasoenteric tube (Figure 2) will then inserted through the nose. Once it has passed the stomach and the first part of the small intestine, the balloon at the terminal end will be inflated to approximately $6 \mathrm{ml}$ and will be used to carry the tube through the small intestine by peristalsis. The tube position will be confirmed by fluoroscopy throughout the tube insertion process. Dilute barium sulphate may also be administered in order to help confirm the positioning. Once the tube has reached the terminal ileum, the balloon will be deflated and the tube will be restrained from additional movement for the rest of the 4-day visit. Participants will be provided with food and drinks during the tube insertion process. However, the food provided will not be standardised to allow for flexibility to aid tube insertion. Following the tube insertion, participants will travel back to the NIHR Imperial CRF, Hammersmith Hospital, accompanied by a trained medical professional. The final position of the tube will be recorded in the participant's notes.

Day 1 - Start of dietary intervention. Participants will be fed one of the three diets over the 4-day study period according to their randomisation. The dietary intervention will start with dinner on Day 1 and end with lunch on Day 4. Meals will be provided at set times; breakfast at 09:00, lunch at 13:00, dinner at 17:00 and an evening snack at 21:00. During their stay, participants will be asked to eat all of the food that is provided and to not eat any other food including chewing gum. Participants will have free access to water, except for during sampling periods on Day 3 and 4 where water will be limited. Participants will not be allowed to leave the CRF during their visit but will have access to $\mathrm{WiFi}$ and TV. Participants will be asked to collect a stool sample each time they pass stool during their inpatient stay for metabolomic and microbiological analysis.

Day 2 - Acclimatisation day. Day 2 will allow participants to acclimatise to their new diet and environment. Meals will be provided at set meal times and participants will have free access to water. The only samples collected on Day 2 will be stool samples.

Day 3 - Ileal sampling. On day 3, ileal samples will be collected via the nasoenteric tube for metabolomic and microbiological analysis. For the 30 minutes before breakfast, the participant's water jug will be removed. Two baseline ileal samples will be collected $>10 \mathrm{~min}$ apart. Breakfast will be served at 9:00 am $( \pm 10 \mathrm{~min})$ with $500 \mathrm{ml}$ water. The time at which each participant starts eating breakfast will be considered to be $0 \mathrm{~min}$. Further ileal samples will be collected every $60 \mathrm{~min}$ for $480 \mathrm{~min}$. Lunch will be served directly after the $240 \mathrm{~min}$ sample with $500 \mathrm{ml}$ water. Dinner will be served at 17:00, after the $480 \mathrm{~min}$ sample and participants will have free access to water for the rest of the day.

Day 4 - Ileal and blood sampling. On the morning of day 4, participants will have an intravenous cannula inserted to allow for blood sampling and body composition will be assessed by bio-impedance analysis. For the 30 minutes before breakfast, the participant's water jug will be removed, as on day 3. Two baseline samples will be collected $>10 \mathrm{~min}$ apart before breakfast. At each timepoint, subjective feelings of appetite ('how hungry/ full do you feel?') and mood ('how nauseous do you feel?') will be collected using a series of $100 \mathrm{~mm}$ visual analogue scales (VAS). The left extremity of the VAS is labelled with 'not at all' and the right-hand extremity is labelled with 'extremely'. Participants will be asked to draw a vertical line on the VAS depending on how intensely they are experiencing each feeling. Baseline breath $\mathrm{H}_{2}$ measurements will be collected in realtime using a handheld monitor (Gastro+ Gastrolyser Breath Hydrogen Monitor, Bedfont Scientific) and will be used as a marker of intestinal fermentation ${ }^{24}$. Baseline blood samples will be collected to assess blood hormones and metabolites and ileal samples will be collected via the nasoenteric tube as on Day 3 . Ileal samples will be collected across both Day 3 and 4 in order to gain a full understanding of the dynamic change of the ileum 
environment over the study period. Once both baseline samples have been collected, breakfast will be served at 9:00 am $( \pm 10 \mathrm{~min})$ with $500 \mathrm{ml}$ water. The time at which the participant starts eating breakfast will be considered to be $0 \mathrm{~min}$. Further samples will be collected every $60 \mathrm{~min}$ for $480 \mathrm{~min}$. Lunch will be served directly after the $240 \mathrm{~min}$ sample with $500 \mathrm{ml}$ water. Following the final sample at $480 \mathrm{~min}$, the cannula and nasoenteric tube will be removed and participants will be discharged.

\section{Sample preparation}

Stool samples. Stool samples will be frozen at $-80^{\circ} \mathrm{C}$ immediately following collection and the time of collection will be recorded.

Ileal samples. In order to collect ileal samples, the aspiration channel of the tube will first be flushed with a volume of water that is equal to the deadspace leading to the lumen. The air channel will be opened and the volume of water that the tube was flushed with will be taken back using a $50 \mathrm{ml}$ syringe. Once the flush has been collected, the aspiration channel will be clamped using a medical clamp and the flush will be discarded. Up to $5 \mathrm{ml}$ ileal sample will be collected at each sampling timepoint. Samples will be put on ice immediately following collection and frozen at $-80^{\circ} \mathrm{C}$. Following sampling, the aspiration channel of the tube will be flushed with a volume of water that is equal to the deadspace and both the aspiration and air channels will be closed until the next sample.

Blood samples. A total of $10 \mathrm{ml}$ blood will be collected at each time point and aliquoted into vacutainers: $1 \mathrm{ml}$ blood will be added to a BD Fluoride Ethylenediaminetetraacetic acid (EDTA) Vacutainer; $2 \mathrm{ml}$ blood will be added to a BD Lithium Heparin Vacutainer containing $20 \mu \mathrm{l} / \mathrm{ml}$ whole blood Aprotinin pancreatic protease inhibitor (Nordic Pharma UK Ltd, Reading, UK), $2 \mathrm{ml}$ blood will be added to a BD Lithium Heparin Vacutainer and $5 \mathrm{ml}$ blood will be added to a BD Serum SST Vacutainer. Plasma tubes will be centrifuged immediately at 2,500 relative centrifugal force $(\mathrm{RCF})$ for $10 \mathrm{~min}$ at $4^{\circ} \mathrm{C}$. Serum tubes will be allowed to clot before centrifugation. Resulting plasma and serum will be separated and frozen at $-80^{\circ} \mathrm{C}$ until analysis.

\section{Sample analysis}

Samples will be analysed upon completion of the study. Metabolic and microbiological profiling of ileal and faecal samples will be performed using ${ }^{1} \mathrm{H}-\mathrm{NMR}$ spectroscopy and $16 \mathrm{~S}$ sequencing, respectively. The metabolic and hormonal profiling of blood samples will be assessed using ${ }^{1} \mathrm{H}-\mathrm{NMR}$ spectroscopy, radioimmunoassays, Ultraperformance Liquid Chromatograpy coupled to Mass Spectrometry (UPLC-MS) and Gas Chromatography coupled to Mass Spectrometry (GC-MS). Subjective appetite will be assessed using visual analogue scales (VAS) and breath $\mathrm{H}_{2}$ will be measured in real-time using a handheld gastrolyser.

\section{In-vitro continuous fermentation model system}

A one-stage continuous fermentation model system will be established using ileal samples from the human study to monitor in vitro the effect of carbohydrate quality on ileal microbiota composition and their subsequent metabolites. The system will be adapted from the three-stage colonic model described by Gibson et al. ${ }^{25}$. Ileal samples will be collected via a nasoenteric tube (Figure 2) as previously described and anaerobically transferred into hungate tubes, which will then be used to inoculate the in vitro system. Samples will be obtained from the system at baseline $(\mathrm{t}=0)$ and 24 hours after $(\mathrm{t}=24)$.

The diets from the human study will then be tested in the in vitro system. Diets will be digested by an in vitro simulation of upper gut digestion as per Mills et al. ${ }^{26}$. The remaining products will be dialysed using a membrane of 100-200 Dalton cut off (Biotech CE Dialysis Tubing, Spectrum Europe, Netherlands). The products will be freeze-dried before their addition to the in vitro system. Samples will be obtained from the system 24 hours after the addition of the diets $(\mathrm{t}=48)$ to measure main bacterial groups using fluorescent in situ hybridisation (FISH) and for microbial produced metabolites using ${ }^{1} \mathrm{H}-\mathrm{NMR}$ spectroscopy.

\section{Data management and dissemination}

\section{Data management}

Participants will be given an anonymised personal study code number once randomised, which will be used throughout the study and in the analysis of data. The study codes will be kept on departmental databases. All personal data will be stored in locked filing cabinets in the Section of Investigative Medicine, Imperial College London. Only members of the Section of Investigative Medicine will have access to these. The Principal Investigator and the study team will have access to the final trial dataset.

\section{Statistical methods}

A formal data analysis plan will be drawn up upon completion of the study. However, assuming the data is parametric and conforms to similar studies we have conducted, we would expect our outcome measures to be analysed using a variety of statistical methods. These will include parametric statistics and multivariate analysis using pattern recognition techniques such as principal component analyses (PCA) and partial least squares discriminant analysis (PLS-DA) and non-metric multidimensional scaling (NMDS) plots, using a number of statistical packages including SPSS, MATLAB and R.

\section{Data monitoring}

As this is a pilot study, there are too few participants to have a data monitoring committee.

\section{Trial sponsor}

Imperial College is the main research sponsor for this study.

\section{Dissemination of results}

Once data analysis is complete, participants will receive a lay summary of the study's findings. In addition, the results of the study will be presented at scientific meetings and conferences, and published in relevant high-impact journals. Research papers will be written by the study team. Authors will be included according to the International Committee of Medical Journal 
Editors (ICMJE) Recommendations. An anonymised dataset will be made available to the scientific community.

\section{Ethical approval}

This study has been approved by the London - Bloomsbury Research Ethics Committee (REC) and Health Research Authority (HRA; REC Reference Number: 17/LO/0354). This study will be conducted in accordance with the recommendations for physicians involved in research on human subjects adopted by the $18^{\text {th }}$ World Medical Assembly, Helsinki 1964 and later revisions. Any substantial amendments made to the protocol, PIS or consent form will be submitted to the REC and HRA. Once these changes have been approved, these modifcations will be explained to participants and implemented with their consent.

Participants will have the choice during the consent process to agree to their samples being stored and used for future analyses. Samples will be kept in the Section of Investigative Medicine and will only be used for other research purposes that have been ethically approved.

\section{Adverse events}

Any significant adverse event as assessed by the researchers will halt the study and the research ethics committee and sponsor will be informed as per standard protocol. All adverse events will be recorded and investigators will review each adverse event as it arises.

\section{Indemnity}

Imperial College London holds negligent harm and non-negligent harm insurance policies, which apply to this study.

\section{Discussion}

The maintenance of body weight throughout life is important for metabolic homeostasis. Over the last 15 years, both epidemiological and experimental evidence have highlighted the importance of carbohydrate fermentation within the gut to appetite regulation. However, our current investigative tools are not capable of characterising the complex signalling that mediates these effects in the gut. In the current study, we will investigate how the quality of dietary carbohydrate consumed affects the form and type of carbohydrate in the ileum. This will allow us to gain a deeper understanding of the composition of the digesta reaching the colon from the ileum following such diets. In addition, we will determine how this subsequently affects subjective appetite measures and gut hormone secretion. We will use an in vitro model, inoculated with ileal fluid, to provide a deeper understanding of how ileum contents, following diets differing in carbohydrate quality, affects the colonic gut microbiota and the production of SCFAs in the colon. This is important in identifying how dietary carbohydrate drives colonic gut hormone release and is critical in understanding the relationship between the colon and the maintenance of energy homeostasis. This knowledge may facilitate the development of food products designed for body weight maintenance.
Study progress

The first study visit was completed in October 2017. Eight participants have successfully completed the study so far.

\section{Data availability}

Underlying data

No underlying data are associated with this article.

\section{Extended data}

Figshare: A study protocol for a randomised crossover study evaluating the effect of diets differing in carbohydrate quality on ileal content and appetite regulation in healthy humans. https://doi.org/10.6084/m9.figshare.7752131.v123.

This project contains the following extended data:

- Participant Information Sheet - Version 5 - 111017.doc (information sheet for research participants)

- Consent Form - Version 1 - 060217.doc (consent form given to each participant)

\section{Reporting guidelines}

Figshare: SPIRIT checklist for "A study protocol for a randomised crossover study evaluating the effect of diets differing in carbohydrate quality on ileal content and appetite regulation in healthy humans". https://doi.org/10.6084/m9.figshare.7752131.v123.

Data are available under the terms of the Creative Commons Attribution 4.0 International license (CC-BY 4.0).

\section{Grant information}

This article presents independent research funded by the UK Biotechnology \& Biological Sciences Research Council (BBSRC) (BB/N016847/1) and Nestec Ltd, and is supported by the NIHR CRF and BRC at Imperial College Healthcare NHS Trust. The views expressed are those of the authors and not necessarily those of our funders, the NHS, the NIHR or the Department of Health. The Section of Endocrinology and Investigative Medicine is funded by grants from the MRC, BBSRC, NIHR, an Integrative Mammalian Biology (IMB) Capacity Building Award, an FP7- HEALTH- 2009- 241592 EuroCHIP grant and is supported by the NIHR Biomedical Research Centre Funding Scheme. GF holds an NIHR Senior Investigator Award.

The funders had no role in study design, data collection and analysis, decision to publish, or preparation of the manuscript.

\section{Acknowledgements}

We would like to acknowledge the support and help of our colleagues in the NIHR Imperial CRF and the Charing Cross Imaging Unit. 
1. WHO: Obesity and overweight - Fact sheet at World Health Organisation Media Centre 2016.

Reference Source

2. Peeters A, Barendregt JJ, Willekens $\mathrm{F}$, et al:: Obesity in adulthood and its consequences for life expectancy: a life-table analysis. Ann Intern Med. 2003; 138(1): 24-32.

PubMed Abstract | Publisher Full Text

3. Butland B, Jebb S, Kopelman P, et al:: Tackling Obesities: Future Choices Project Report, 2nd Edition. 2007. Contract No.: 17 March. Reference Source

4. Hill JO, Peters JC: Biomarkers and functional foods for obesity and diabetes. Br J Nutr. 2002; 88 Suppl 2: S213-S8. PubMed Abstract | Publisher Full Text

5. Murphy KG, Bloom SR: Gut hormones in the control of appetite. Exp Physiol. 2004; 89(5): 507-16.

PubMed Abstract | Publisher Full Text

6. Abbott CR, Monteiro M, Small CJ, et al:: The inhibitory effects of peripheral administration of peptide $\mathrm{YY}_{3-36}$ and glucagon-like peptide-1 on food intake are attenuated by ablation of the vagal-brainstem-hypothalamic pathway. Brain Res. 2005; 1044(1): 127-31.

PubMed Abstract | Publisher Full Text

7. Flint A, Raben A, Astrup A, et al:: Glucagon-like peptide 1 promotes satiety and suppresses energy intake in humans. J Clin Invest. 1998; 101(3): 515-20. PubMed Abstract | Publisher Full Text | Free Full Text

8. Batterham RL, Cohen MA, Ellis SM, et al:: Inhibition of food intake in obese subjects by peptide YY. . N Engl J Med. 2003; 349(10): 941-8. PubMed Abstract | Publisher Full Text

9. Batterham RL, Cowley MA, Small CJ, et al.: Gut hormone $\mathrm{PYY}_{3-36}$ physiologically inhibits food intake. Nature. 2002; 418(6898): 650-4.

PubMed Abstract | Publisher Full Text

10. Challis BG, Pinnock SB, Coll AP, et al:: Acute effects of PYY $Y_{3-36}$ on food intake and hypothalamic neuropeptide expression in the mouse. Biochem Biophys Res Commun. 2003; 311(4): 915-9. PubMed Abstract | Publisher Full Tex

11. Liu S, Willett WC, Manson JE, et al:: Relation between changes in intakes of dietary fiber and grain products and changes in weight and development of obesity among middle-aged women. Am J Clin Nutr. 2003; 78(5): 920-7. PubMed Abstract | Publisher Full Text

12. Ludwig DS, Pereira MA, Kroenke $\mathrm{CH}$, et al.: Dietary fiber, weight gain, and cardiovascular disease risk factors in young adults. JAMA. 1999; 282(16): 1539-46.

PubMed Abstract | Publisher Full Text

13. Byrne CS, Chambers ES, Morrison DJ, et al:: The role of short chain fatty acids in appetite regulation and energy homeostasis. Int J Obes (Lond). 2015; 39(9): $1331-8$.

PubMed Abstract | Publisher Full Text | Free Full Text
14. Slavin JL: Dietary fiber and body weight. Nutrition. 2005; 21(3): 411-8. PubMed Abstract | Publisher Full Text

15. Grundy MM, Edwards $\mathrm{CH}$, Mackie AR, et al: Re-evaluation of the mechanisms of dietary fibre and implications for macronutrient bioaccessibility, digestion and postprandial metabolism. Br J Nutr. 2016; 116(5): 816-33. PubMed Abstract | Publisher Full Text | Free Full Text

16. Berry SE, Tydeman EA, Lewis HB, et al.: Manipulation of lipid bioaccessibility of almond seeds influences postprandial lipemia in healthy human subjects. Am J Clin Nutr. 2008; 88(4): 922-9.

PubMed Abstract | Publisher Full Tex

17. Edwards $\mathrm{CH}$, Grundy MM, Grassby $\mathrm{T}$, et al.: Manipulation of starch bioaccessibility in wheat endosperm to regulate starch digestion, postprandial glycemia, insulinemia, and gut hormone responses: a randomized controlled trial in healthy ileostomy participants. Am J Clin Nutr. 2015; 102(4): 791-800. PubMed Abstract | Publisher Full Text | Free Full Text

18. Gidley MJ: Hydrocolloids in the digestive tract and related health implications. Curr Opin Colloid Interface Sci. 2013; 18(4): 371-8. Publisher Full Text

19. Murphy KG, Bloom SR: Gut hormones and the regulation of energy homeostasis. Nature. 2006; 444(7121): 854-9. PubMed Abstract | Publisher Full Text

20. Spreckley E, Murphy KG: The L-Cell in Nutritional Sensing and the Regulation of Appetite. Front Nutr. 2015; 2: 23. PubMed Abstract | Publisher Full Text | Free Full Text

21. Flint $\mathrm{HJ}$, Scott KP, Louis $P$, et al.: The role of the gut microbiota in nutrition and health. Nat Rev Gastroenterol Hepatol. 2012; 9(10): 577-89. PubMed Abstract | Publisher Full Text

22. Cummings $\mathrm{JH}$, Pomare EW, Branch WJ, et al: : Short chain fatty acids in human large intestine, portal, hepatic and venous blood. Gut. 1987; 28(10): 1221-7. PubMed Abstract | Publisher Full Text | Free Full Text

23. Byrne C, Blunt D, Burn J, et al:: A study protocol for a randomised crossover study evaluating the effect of diets differing in carbohydrate quality on ileal content and appetite regulation in healthy humans. figshare. Fileset. 2019 http://www.doi.org/10.6084/m9.figshare.7752131.v1

24. Levitt MD: Production and excretion of hydrogen gas in man. N Engl J Med. 1969; 281(3): 122-7.

PubMed Abstract | Publisher Full Text

25. Gibson GR, Cummings JH, Macfarlane GT: Use of a three-stage continuous culture system to study the effect of mucin on dissimilatory sulfate reduction and methanogenesis by mixed populations of human gut bacteria. Appl Environ Microbiol. 1988; 54(11): 2750-5. PubMed Abstract | Free Full Text

26. Mills DJ, Tuohy KM, Booth J, et al.: Dietary glycated protein modulates the colonic microbiota towards a more detrimental composition in ulcerative colitis patients and non-ulcerative colitis subjects. J Appl Microbiol. 2008; 105(3): 706-14.

PubMed Abstract | Publisher Full Text 


\title{
Open Peer Review
}

\section{Current Peer Review Status: ? ?}

\section{Version 1}

Reviewer Report 23 September 2019

https://doi.org/10.5256/f1000research.19542.r47113

(C) 2019 Schellekens $\mathbf{H}$ et al. This is an open access peer review report distributed under the terms of the Creative Commons Attribution License, which permits unrestricted use, distribution, and reproduction in any medium, provided the original work is properly cited.

\author{
Marcel van de Wouw \\ APC Microbiome Ireland, University College Cork, Cork, Ireland \\ Harriët Schellekens \\ ${ }^{1}$ APC Microbiome Ireland, University College Cork, Cork, Ireland \\ 2 Department of Anatomy \& Neuroscience, University College Cork, Cork, Ireland
}

The study protocol submitted by Byrne et al. is an exciting and well-thought out experiment, aimed at investigating the impact of diets with different carbohydrate quality (i.e. low fibre, whole high fibre and disrupted high fibre) on the gastrointestinal microbiota and appetite in humans. The repeated ileal sampling provides for a real time analysis of the response of the microbiota to these different diets.

\section{Minor comments:}

Some lines of research indicate that different dietary fibres (e.g. inulin, rhamnose) differentially impact the gut microbiota and subsequent SCFA production (Baxter et al. 2019 1; Reichardt et al. 2018²). This is well-controlled for when comparing the HF-whole and HFdisrupted groups, but not so much for the LF-refined group. It might be good to, if possible, analyse dietary fibre profile of the diets. This will increase the interpretability of the data and allow for a better comparison between the LF and HF groups, as LF will likely have a different dietary fibre profile.

It is likely that the food intake patterns of participants prior to the study will influence their microbiota and therefore how their microbiota will respond to the different diets. Performing a food frequency questionnaire to assess their diet prior to the visit date might therefore provide valuable insight into how day-to-day food intake patterns can influence the microbiota and its response to these diets.Or any other measure to have an record of baseline food intake and food quality.

Both males and females will be recruited, but its not stated if the aim is to recruit at a gender balanced level. Use of oral contraceptives and menstrual cycle should be recorded (oral contraceptives possibly excluded). 
Page 3, sentence: "Short-chain fatty acids, products of microbial fermentation, have also been shown to stimulate the secretion of GLP-1 and PYY." This sentence requires a reference.

\section{References}

1. Baxter N, Schmidt A, Venkataraman A, Kim K, et al.: Dynamics of Human Gut Microbiota and Short-Chain Fatty Acids in Response to Dietary Interventions with Three Fermentable Fibers. mBio. 2019; 10 (1): e02566-18 Publisher Full Text

2. Reichardt N, Vollmer M, Holtrop G, Farquharson FM, et al.: Specific substrate-driven changes in human faecal microbiota composition contrast with functional redundancy in short-chain fatty acid production.ISMEJ. 12 (2): 610-622 PubMed Abstract | Publisher Full Text

Is the rationale for, and objectives of, the study clearly described?

Yes

Is the study design appropriate for the research question?

Yes

Are sufficient details of the methods provided to allow replication by others? Partly

Are the datasets clearly presented in a useable and accessible format? Not applicable

Competing Interests: No competing interests were disclosed.

Reviewer Expertise: Microbiota-gut-brain axis in metabolism and eating behaviour

We confirm that we have read this submission and believe that we have an appropriate level of expertise to confirm that it is of an acceptable scientific standard, however we have significant reservations, as outlined above.

Author Response 11 Oct 2019

Aygoul Dagbasi, Imperial College London, London, UK

Thank you for your time to review our article and provide valuable comments.

We agree with all your comments.

We will be performing a direct analysis of the dietary fibre profile of each dietary intervention to be able to better interpret the data as suggested. As you highlighted, high fibre groups (whole and blended) will have the same profiles since these diets consist of the same foods however the low fibre group will have a different profile. 
The cross-over design of the study allows each participant to act as their own control. This will eliminate confounding factors like habitual dietary intake and gut microbiota profile. Current dietary assessment methods provide an inaccurate measure of dietary intake (Shim et al. 2014). As a result of these; we decided not to collect dietary intake data. We will be excluding participants following extreme diets such as vegan or vegetarian diets.

We will aim to recruit at a gender-balanced level. We will note information regarding females' contraception methods and menstrual cycles.

Thank you again for your valuable comments

Competing Interests: No competing interests were disclosed.

Reviewer Report 05 September 2019

https://doi.org/10.5256/f1000research.19542.r45303

(C) 2019 Blaak E. This is an open access peer review report distributed under the terms of the Creative Commons Attribution License, which permits unrestricted use, distribution, and reproduction in any medium, provided the original work is properly cited.

\section{Ellen E. Blaak}

Department of Human Biology, NUTRIM School of Nutrition and Translational Research in Metabolism, Maastricht University Medical Centre, Maastricht, The Netherlands

This is an interesting study design investigating the role of the ileum and its microbiota in the processing of dietary fibers on 3 different diet, a low fiber diet and 2 high fiber diets, one with intact cellular structures and the other one with disrupted cell structures. This study gives a real time perspective how the ileum content changes in response to different diets and is therefore unique.

The hypothesis may be formulated a little bit sharper, the idea is that minimal processed fiber may lead to a higher fermentation in the colon with a more beneficial metabolic profile, how exactly is this question answered since fermentation and SCFA production is not directly measured in the colon?

Would an increased fermentation in the ileum also be beneficial?

How much time does the sampling of the ileum take? Is also the total microbial content determined in ileal and fecal samples, expressed per what?

What is the period between the visits i.e. the washout period?

The in vitro approach may be an interesting addition. Sampling takes place at baseline and at $24 \mathrm{~h}$, why are these time points chosen and is more frequent sampling a possibility? 
Looking at the effect of whole diets in the 'in vitro' system, diets are first freeze dried, to what extent are the cellular structures maintained in this procedure and to what extent are the outcomes still relevant for the in vivo conditions?

Overall, a challenging and interesting study protocol.

Is the rationale for, and objectives of, the study clearly described?

Yes

Is the study design appropriate for the research question?

Yes

Are sufficient details of the methods provided to allow replication by others? Partly

Are the datasets clearly presented in a useable and accessible format? Not applicable

Competing Interests: No competing interests were disclosed.

Reviewer Expertise: metabolic interorgan crosstalk in insulin resistance

I confirm that I have read this submission and believe that I have an appropriate level of expertise to confirm that it is of an acceptable scientific standard, however I have significant reservations, as outlined above.

Author Response 11 Oct 2019

Aygoul Dagbasi, Imperial College London, London, UK

Thank you for your time to review our article and provide valuable comments.

We agree with all your comments.

We will be changing our hypothesis as per your advice to fit the study design better.

We do think that an increased fermentation in the ileum would also be beneficial in addition to the colonic fermentation. This is because GLP-1 and PYY secreting L-cells increase in number along the GI tract with the highest numbers found in distal ileum and colon (Suzuki et al., 2018). Therefore the composition of the ileal microbiota and their subsequent metabolites including SCFAs can be sensed by the dense population of ileal L-cells and potentially have a profound effect on appetite hormone secretion and host metabolism. We are hoping to answer this question better with our study results.

We had a trial run of ileal sampling which took around 5-10 minutes per sample. The microbial content will be expressed as Log 10 numbers per $\mathrm{ml}$ of ileal/faecal content as 
measured by flow-FISH (fluorescent in-situ hybridisation) and as ratios when measured by 16 s rRNA sequencing.

The washout period will be minimum of 7 days.

The in-vitro system takes 24 hours to stabilise after inoculation and after the addition of diets therefore any samples taken in between would be inaccurate. Sampling can continue after 24 hours however since the system is stabilised, no change is anticipated after this period.

Microscopy pictures were taken after the diets were freeze dried. These revealed that this method is not disruptive to the cellular structures. The slides were stained with iodine which showed carbohydrate still present in intact cellular structures for Diet 2 (Whole Diet).

Thank you again for your valuable comments.

Competing Interests: None

The benefits of publishing with F1000Research:

- Your article is published within days, with no editorial bias

- You can publish traditional articles, null/negative results, case reports, data notes and more

- The peer review process is transparent and collaborative

- Your article is indexed in PubMed after passing peer review

- Dedicated customer support at every stage

For pre-submission enquiries, contact research@f1000.com 